\title{
Universal 3-Dimensional Visibility Representations for Graphs
}

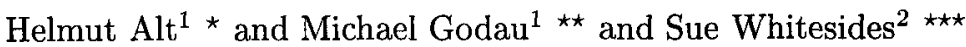 \\ 1 Freie Universität Berlin Berlin, Germany \\ 2 McGill University Montreal, Canada
}

\begin{abstract}
This paper studies 3-dimensional visibility representations of graphs in which objects in 3-d correspond to vertices and vertical visibilities between these objects correspond to edges. We ask which classes of simple objects are universal, i.e. powerful enough to represent all graphs. In particular, we show that there is no constant $k$ for which the class of all polygons having $k$ or fewer sides is universal. However, we show by construction that every graph on $n$ vertices can be represented by polygons each having at most $2 n$ sides. The construction can be carried out by an $O\left(n^{2}\right)$ algorithm. We also study the universality of classes of simple objects (translates of a single, not necessarily polygonal object) relative to cliques $K_{n}$ and similarly relative to complete bipartite graphs $K_{n, m}$.
\end{abstract}

\section{Introduction}

This paper considers 3-dimensional visibility representations for graphs. Vertices are represented by 2-dimensional objects floating in 3-d parallel to the $x y$-plane (these objects can be swept in the $z$ direction to form thick objects if desired). There is an edge in the graph if, and only if, the objects corresponding to its endpoints can see each other along a thick line of sight parallel to the $z$-axis. A thick line of sight is a tube of arbitrarily small by positive radius whose ends are contained in the objects. Throughout this paper, we use the term "visibility representation" to refer to this particular model.

The corresponding notion of 2-dimensional visibility has received wide attention due to its applications to such areas as graph drawing, VLSI wire routing, algorithm animation, CASE tools and circuit board layout. See [DETT] for a survey on graph drawing in general; for 2-dimensional visibility representations, see for example [DH], [TT], [KKU], [W].

Exploration of 3-dimensional visibility is still in the early stages. From the point of view of geometric graph theory, it is natural to consider visibility representations of graphs in dimensions higher than 2. From the point of view of

* alt@inf.fu-berlin.de Institut für Informatik, FU Berlin, Takustr. 9, 14195 Berlin, Germany. This research was supported by the ESPRIT Basic Research Action No. 7141, Project ALCOM II.

$\star \star$ godau@inf.fu-berlin.de

$\star \star \star$ sue@cs.mcgill.ca Written while the author was visiting INRIA-Sophia Antipolis and Freie Universität Berlin. Research supported by NSERC and FCAR grants. 
visualization of graphs, it is basic to ask whether 3-dimensional representations give useful visualizations. For a 3-dimensional representation to be useful for visualization, it should be powerful enough to represent all graphs, or at least basic kinds of graphs. This motivates us to ask which classes of objects are universal, i.e., can give visibility representations for all graphs, or all graphs of a given kind?

The visibility representation considered in this paper has also been studied in [BEF+] (an abstract was presented at GD'92), in [Rom], and in [FHW]. In these papers, the objects representing vertices are axis-aligned rectangles, or disks, and the properties of graphs that can be represented by these objects are studied. By contrast, this paper begins with families of graphs (all graphs, or all graphs of a specific kind), and explores simple ways to represent all graphs in the family.

Section 2 considers which translates of a given, fixed figure are universal for cliques $K_{n}$ and complete bipartite graphs $K_{m, n}$. Section 3 uses counting arguments based on arrangements to show that no class of polygons having at most some fixed number $k$ of sides is strong enough to represent all graphs. Section 4 shows that every graph on $n$ vertices has a visibility representation by polygons each of which has at most $2 n$ sides. These sections also contain additional results not listed here in the introduction.

\section{Graphs realizable by translates of a figure}

In this section we will investigate which complete and which complete bipartite graphs can be realized as visibility graphs of translates of one fixed figure. Here a figure is defined as an open bounded set whose boundary is a Jordan-curve. We say that a graph $G$ can be realized by a figure $\mathrm{F}$ iff $G$ is the visibility graph of translates of $F$. It will turn out, for example, that with many figures arbitrary complete graphs can be realized whereas each figure can only realize a finite number of stars, i.e. complete bipartite graphs of the form $K_{1, n}$.

\subsection{Complete graphs}

The realization of complete graphs $K_{n}$ by translates of special figures like squares and disks has been investigated by Fekete, Houle, and Whitesides [FHW] and by Bose et al. $[\mathrm{BEF}+]$. In $[\mathrm{FHW}]$ it was shown that $K_{7}$ can be realized by a square, whereas any $K_{n}, n \geq 8$ cannot. On the other hand, any $K_{n}$ can be realized by a disk. We will consider more general figures in the following theorem.

First, we need the following definitions:

A curve $C$ is called strictly convex, iff for any two points $p, q \in C$ the interior of the line segment $\overline{p q}$ does not intersect $C$. We say that a figure $F$ has a local roundness if there is some open set $U$ such that $U \cap \partial F$ is a strictly convex curve.

Theorem 2.1 a) Any $K_{n}$ can be realized by any nonconvex polygon.

b) For any convex polygon $P$ there is an $n \in \mathbb{N}$ such that no $K_{m}, m \geq n$ can be realized by $P$. 
c) To any $K_{n}$ there is a convex polygon realizing it.

d) Any figure $F$ with a local roundness can realize any $K_{n}$.

Proof. a)We first observe that the figure in Fig.1 can realize any $K_{n}$. If $P$ is a nonconvex polygon then it has at least one nonconvex vertex. Arranging copies of $P$ in a neighborhood of this vertex as in Fig. 1 realizes any $K_{n}$. b)(Sketch)

Let $P_{1}, \ldots, P_{k}$ be a sequence of (projections of) translates of a convex $n$-gon ordered by increasing $z$-coordinates, $e_{1}, \ldots, e_{k}$ the corresponding translates of one edge, and $H_{i}$ the halfplane bounded by the straight line through $e_{i}$ which contains $P_{i}, i=1, \ldots, k$. We define a linear order on $e_{1}, \ldots, e_{k}$ (more precisely, on the set of lines passing through them) by: $e_{i} \leq e_{j} \Longleftrightarrow H_{i} \supseteq H_{j}$. By geometric considerations it can be shown:

Claim: If $P_{1}, P_{2}, P_{3}$ are translates of a convex polygon realizing $K_{3}$, then not all sequences $e_{1}, e_{2}, e_{3}$ of translates of one edge can be monotone in the above order.

For example in Fig. $2 e_{1}, e_{2}, e_{3}$ is monotone increasing, $d_{1}, d_{2}, d_{3}$ monotone decreasing, but $c_{1}, c_{2}, c_{3}$ is not monotone.

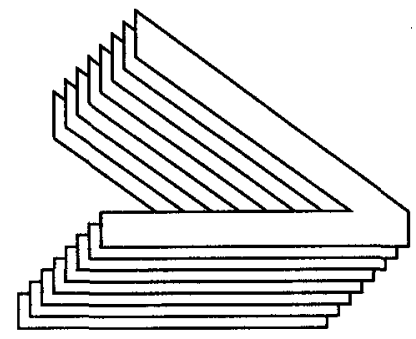

Fig. 1. Realization of an arbitrary $K_{n}$ with a nonconvex polygon

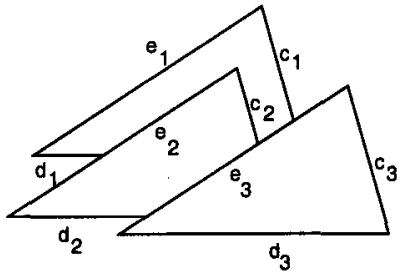

Fig. 2. Triangles realizing $K_{3}$.

Now let $f(k)=(k-1)^{2}+1$ for $k \in \mathbb{N}$ and let for $n \in \mathbb{N} N:=f^{n}(3)(n$ fold iteration of $f$; actually $N=2^{2^{n}}+1$ ). Using an argument from [BEF+] we will show that $K_{N}$ cannot be realized by any convex $n$-gon. Suppose otherwise and let $e^{1}, \ldots, e^{n}$ be the edges and $P_{1}, \ldots, P_{N}$ the translates of the $n$-gon. Since $N=\left(f^{n-1}(3)-1\right)^{2}+1$ by the Theorem of Erdös-Szekeres [ES] the sequence $e_{1}^{1}, \ldots, e_{N}^{1}$ of translates of edge $e^{1}$ has a monotone subsequence of length $f^{n-1}(3)$. Considering the corresponding subsequence of polygons it must have a subsequence of length $f^{n-2}(3)$ where both the $e^{1}$ - and $e^{2}$-sequences are monotone. Iterating this process we would obtain a subsequence of length $f^{0}(3):=3$ where all edge-sequences are monotone in contradiction to the claim above. c) follows

from the fact that any $K_{n}$ can be realized by disks and any disk can be approximated to arbitrary precision by convex polygons. d) Consider a nondegenerate 
segment of $F^{\prime} s$ boundary that is strictly convex. We can select a suitable subsegment $\sigma$ with the following property: If $l$ is the straight line through $\sigma^{\prime} s$ endpoints then no line perpendicular to $l$ intersects $\sigma$ in more than one point.

Assume also w.l.o.g. that $l$ is horizontal, so $\sigma$ looks as in Fig. 3 .

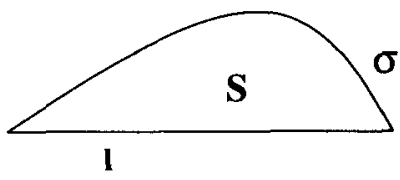

Fig. 3. Curve segment $\sigma$

Let $S$ be the convex figure bounded by $\sigma$ and the line segment between its endpoints. We will show by an inductive construction:

Claim: For any $K_{n}$ there exists a realization by $n$ translates $S_{1}, \ldots, S_{n}$ of $S$ with the following properties:

i) Let $S_{1}^{\prime}, \ldots, S_{n}^{\prime}$ be the projections of $S_{1}, \ldots, S_{n}$ into the $x y$-plane. There exists a horizontal straight line $g$ such that all the horizontal segments of $S_{1}^{\prime}, \ldots, S_{n}^{\prime}$ lie strictly below $g$.

ii) There is a visibility for any pair $S_{i}, S_{j}, i \neq j$ strictly above $g$.

iii) Let $s_{i j}$ be the intersection point of $S_{i}^{\prime}, S_{j}^{\prime}$. For $i=1, \ldots, n-1$ some nondegenerate part $c_{i}$ of $S_{i}$ 's boundary and some part of its interior are visible from $z=\infty$ in any neighborhood of $s_{i, n}$.

iv) The z-coordinate of $S_{i}$ is $i$ for $i=1, \ldots, n$.

The claim is obviously true for $n=1$.

Suppose now by inductive hypothesis that we positioned $S_{1}, \ldots, S_{n}$ satisfying the claim. We choose some point $p$ on the boundary of $S_{n}$ to the right of all $s_{1, n}, \ldots, s_{n-1, n}$ as intersection point $s_{n+1, n}$ (see Fig. 4). Now we position $S_{n+1}$ in the plane $z=n+1$ as follows:

First we put it exactly over $S_{n}$. Then we move it upwards slightly so that i) is still correct. Then it is moved to the left until it intersects $S_{n}$ in $p$ (see Fig. 4). The total motion can be made arbitrarily small, in fact, small enough so that iii) is still satisfied. ii) is satisfied by part iii) of the inductive hypothesis since $s_{n+1}$ covers all points $s_{1, n} \ldots s_{n-1, n}$.

\subsection{Complete Bipartite Graphs}

$[\mathrm{BEF}+]$ considers the realization of complete bipartite graphs by unit disks and unit squares. It is shown that $K_{2,3}$ and $K_{3,3}$ can be realized but $K_{j, 3}, j \geq 4$ cannot. Here we will consider translates of more general convex objects and in particular the realization of stars $K_{1, n}$. In fact, we will show: 


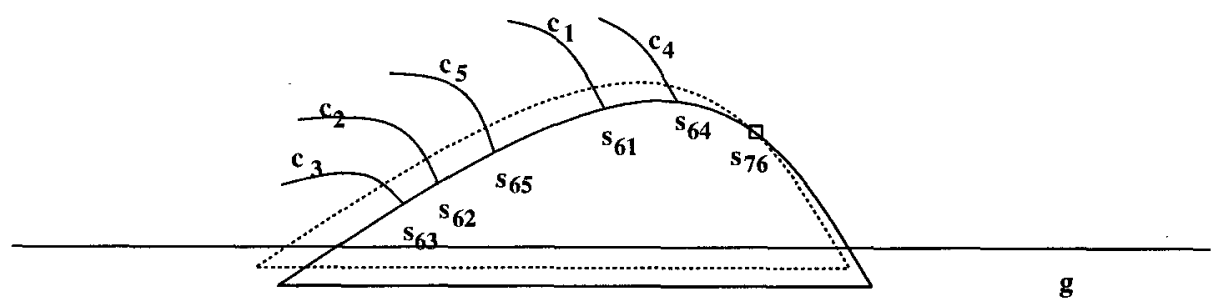

Fig. 4. Construction of $S_{7}$.

Theorem 2.2 a) $K_{1,5}$ but no $K_{1, n}, n \geq 6$ can be realized with parallelograms. b) If $B$ is a strictly convex body $K_{1,6}$ but no $K_{1, n}, n \geq 7$ can be realized by $B$. c) To any figure $F$ there exists an $n \in \mathbb{N}$ such that for all $k \in \mathbb{N}, k \geq n K_{1, k}$ is not realizable by $F$.

d) To any $K_{n, m}$ there exists a quadrilateral realizing it.

Proof. a) A realization of $K_{1,5}$ by parallelograms is quite straightforward. $K_{1, n}$ $n \geq 6$ is not possible since one parallelogram cannot intersect 5 or more disjoint parallelograms of the same size.b) (Sketch) Here we use some results from con-

vexity theory obtained by Hadwiger [H] and Grünbaum [G]. In fact, they showed that at most 8 translates of a convex body $B$ in two dimensions can touch $B$ without intersecting it or each other. The number 8 is only achieved by parallelograms, otherwise it is 6 . For strictly convex bodies we observe that the tangent rays from $B$ separating two neighboring touching translates all point into different directions and their slopes form a strictly monotone sequence (see Figure 5 ). From this it is possible to conclude that if one of the translates is removed one can distribute the others so that they still touch $B$ but not each other any more. Then they can be pushed slightly inward $B$ and we have a realization of $K_{1,5}$. Placing another copy on the other side exactly over $B$ gives a realization of $K_{1,6}$.

The impossibility of $K_{1,7}$ is derived with similar arguments from the fact that no 6 translates of $B$ can intersect $B$ without intersecting each other (see [G]).c) Consider a realization of $K_{1, n}$ and its projection onto the $x y$-plane.

Then no point of the plane can be covered by the projections of more than three of the figures. Furthermore the figure representing the center of the star must be intersected by all others, so all projections must lie within in a circle whose diameter is at most three times the diameter of $F$. These two properties imply that the number of figures is limited.d) The construction is shown in Fig. 6. 


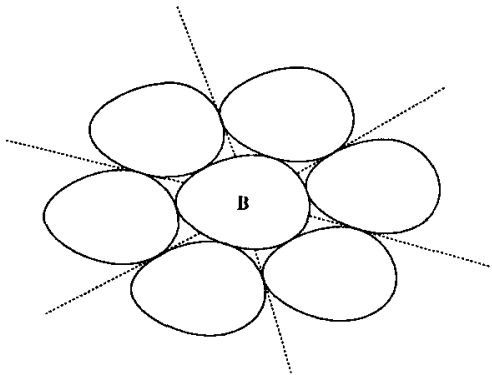

Fig. 5. $B$ touched by 6 of its translates.

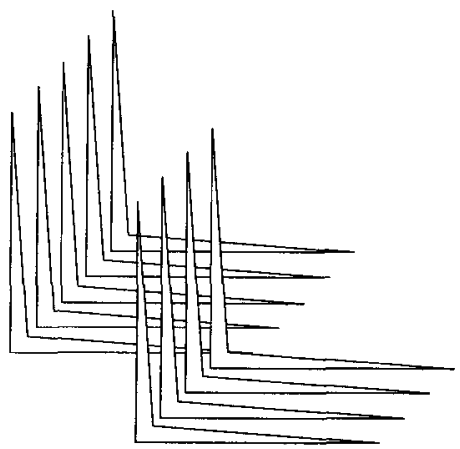

Fig. 6. Realization of $K_{4,5}$ by quadrilaterals

\section{An upper bound on the number of graphs representable by k-gons}

In this section we will show that not each graph has a visibility representation by $\mathrm{k}$-gons for some fixed $k \in \mathbb{N}$. In fact, we will even see that in order to represent all graphs with $n$ vertices by polygons, some of those must have more than $\left\lfloor\frac{\alpha n}{\log n}\right\rfloor$ vertices for some constant $\alpha>0$.

Definition 1. A graph is said to be $k$-representable iff there is a visibility representation with (not necessarily convex) simple polygons each having at most $k$ vertices.

The interesting fact that for every $k$ there is a graph which is not $k$ representable follows from the following theorem.

Theorem 2. There is an $\alpha>0$ and there are graphs $G_{2}, G_{3}, G_{4}, \ldots, G_{n}, \ldots$ such that $G_{n}$ has $n$ vertices and is not $\left\lfloor\frac{\alpha n}{\log n}\right\rfloor$-representable.

The theorem follows quite easily from the following lemma.

Lemma 3. There is a $\beta$ such that for all $n, k$ there can be at most $2^{\beta n k \log (n k)}$ many graphs with a fixed vertex set $V=\left\{v_{1}, \ldots, v_{n}\right\}$ which are $k$-representable.

Proof. We consider an arbitrary $k$-representable graph $G=(V, E)$ with $V=$ $\left\{v_{1}, \ldots, v_{n}\right\}$. Obviously, if $G$ is k-representable then there exists a representation by polygons $P_{1}, \ldots, P_{n}$ parallel to the $x y$-plane with at most $k$ edges each. Without loss of generality we can assume that $P_{i}$ has $z$-coordinate $i$ for $i=1, \ldots, n$.

Consider the projections of all the polygons into the $x y$-plane. Extend each edge $s$ of each polygon to a line $l_{s}$, obtaining a family $\mathcal{L}$ of at most $m:=n k$ not necessarily distinct straight lines. Each edge $s$ and, thus, each line $l_{s}$ can be oriented by the convention that the polygon lies, say, left of $s$. Now, $G$ can be uniquely identified by the information in the following items. 
1. the arrangement of the lines in $\mathcal{L}$.

2. Each polygon $P_{i}, i=1, \ldots, n$ is identified by the description of a counterclockwise tour around its boundary. In particular, the starting point $s$ is given by a line $l \in \mathcal{L}$ containing it and a number $n_{0} \leq m$ meaning that $s$ is the $n_{0}$ th intersection point when traversing $l$ in direction of its orientation. Then a sequence of at most $k$ numbers $n_{1}, \ldots, n_{r} \in\{1, \ldots, m\}$ is given, meaning that the tour starts at $s$, goes straight on $l$ for $n_{1}$ intersections, then turns into the oriented line crossing there, goes straight for $n_{2}$ intersections, etc. Clearly, this describes a tour within the arrangement.

Cleary, the information in the above items uniquely identifies the pairwise intersections of the projections of the polygons into the $x y$-plane. This together with the convention that $P_{i}$ has $z$-coordinate equal to $i$ makes it possible to determine all visibilities, and hence $G$ itself.

It remains to count the number of different possibilities for the data in the above items:

1. As is well known (see [A]) the number of different arrangements of $m$ oriented straight lines is at most $2^{\beta_{1} m \log m}$ for some constant $\beta_{1}>0$.

2. For each polygon there are $m$ possibilities for the starting line $l$, and at most $m$ possibilities for each number $n_{0}, \ldots, n_{r}, r \leq k$. So the number of possibilities per polygon is bounded by $\mathrm{m}^{k+2}$. Altogether, the number of possibilities is at most $m^{(k+2) n}$, which is at most $2^{\beta_{2} m \log m}$ for some constant $\beta_{2}>0$.

Multiplying the upper bounds in 1 and 2 gives the desired total upper bound of $2^{\beta m \log m}$ where $\beta=\beta_{1}+\beta_{2}$.

Since there are exactly $2^{\left(\begin{array}{l}n \\ 2\end{array}\right)}$ graphs with vertex set $V$ there are at least $2^{\left(\begin{array}{l}n \\ 2\end{array}\right)} / n$ ! (pairwise nonisomorphic) graphs with $n$ vertices which is more than $2^{\delta n^{2}}$ for some $\delta>0$. Theorem 2 follows from this lower bound and Lemma 3 .

On the other hand, every graph with $n$ vertices is $(2 n+1)$-representable, which will be shown in the next section.

\section{The Construction}

This section gives a general construction which produces for any graph $G=$ $(V, E)$ a 3-dimensional visibility representation for $G$. The construction can be carried out in a straight-forward manner by an algorithm that runs in $O\left(n^{2}\right)$ time, where $n$ is the number of vertices of $G$. Each vertex is represented by a polygon of $O(n)$ sides (the polygons may differ in shape).

If desired, the basic construction can be modified easily and with the same time complexity to produce convex polygonal (or polyhedral) pieces. Furthermore, these pieces can be made to have all vertex angles of at least $\pi / 6$. By using the technique of [CDR], it is also possible to implement the algorithm in $O\left(n^{2}\right)$ time with respect to a Turing machine model of computation. 


\subsection{The Basic Pieces}

Let $W$ denote a regular, convex $2 n$-gon centered at the origin $O$, and let $w_{1}, w_{2}, \ldots w_{2 n}$ denote the locations of its vertices. We use $W$ to define the basic pieces representing the vertices of $G$. For this purpose, let $X$ denote a regular, convex $n$-gon with vertices located at the odd-indexed vertices of $W$. Imagine adding triangular "tabs" to $X$ to obtain $W$ as follows. Call edge $w_{2 i-1}, w_{2 i+1}$ of $X$ tab position $i$, and for each $i$ from 1 to $n$, add a triangle whose vertices are $w_{2 i-1}, w_{2 i}, w_{2 i+1}$ to $X$ at tab position $i . W$ is $X$ together with its tabs (see Fig. 7).

The pieces of our construction are obtained from $X$ in a similar way, except that the tabs may vary in size. The construction may attach to tab position $i$ of $X$ a tab $T_{i}$ with vertices $w_{2 i-1}, t_{i}, w_{2 i+1}$. Vertex $t_{i}$ is called the tab vertex of $T_{i}$. In general, $T_{i}$ lies inside the corresponding tab on $W$, with vertex $t_{i}$ lying on the radial line through $O$ and $w_{2 i}$.

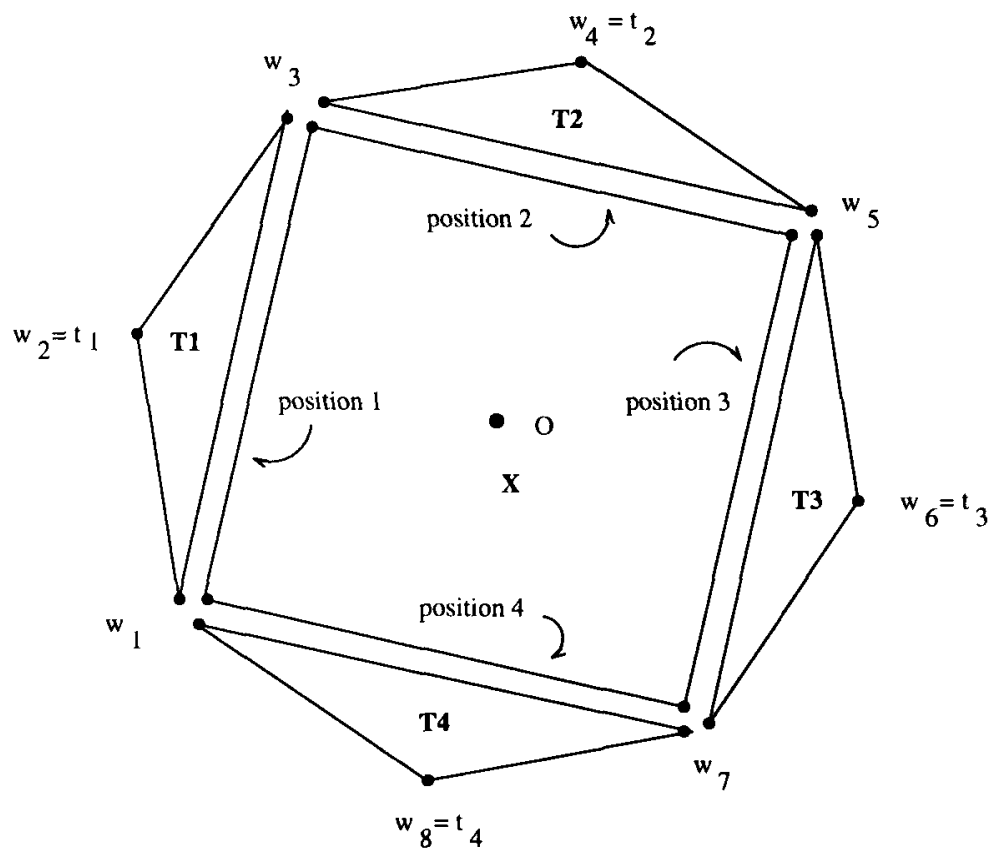

Fig. 7. Regular $n$-gon $X$ for $n=4$ tabs.

Definition 4. Let $p_{2 i}$ denote the point of intersection of the radial line through $O$ and $w_{2 i}$ with the line through $w_{2 i-1}$ and $w_{2 i+1}$. The size $s_{i}$ of tab $T_{i}$ is defined by $s_{i}=n d\left(t_{i}, p_{2 i}\right) / d\left(w_{2 i}, p_{2 i}\right)$.

A tab of full size $n$ has its tab vertex $t_{i}$ positioned at $w_{2 i}$. 
We depth first search $G$, assigning to each vertex a number $i$ indicating the order in which the search discovers the vertex. The $i^{\text {th }}$ vertex discovered is represented by a polygon $P_{i}$ consisting of a wedge-shaped portion of $X$ with tabs of various sizes adjoined. See Fig. 8.

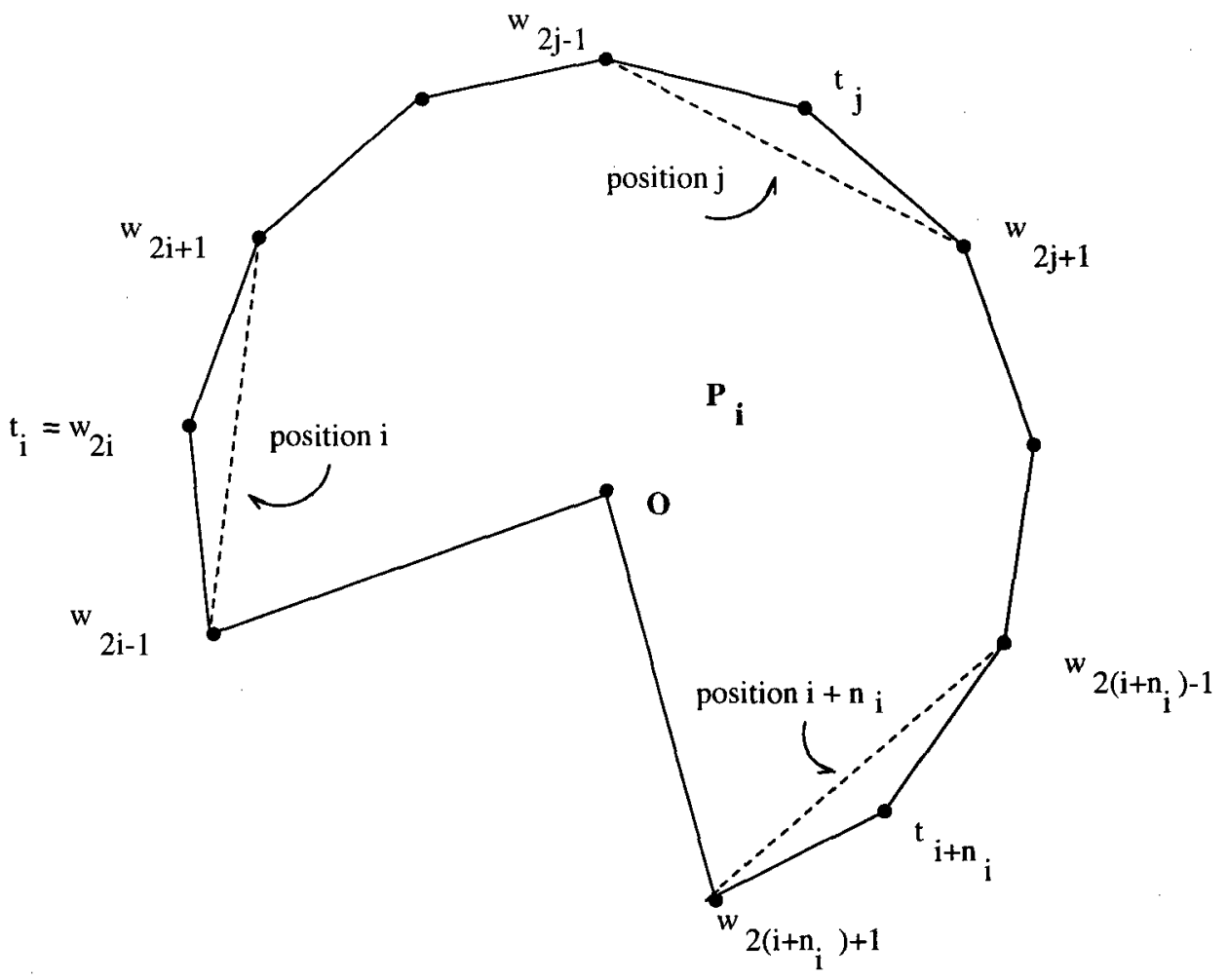

Fig. 8. Piece $P_{i}$.

The bounding wedge of $P_{i}$ is defined by two radial segments emanating from $O$, one to $w_{2 i-1}$ and the other to $w_{2\left(i+n_{i}\right)+1}$, for some $n_{i} \geq 0$ to be determined. Between these radial segments, $X$ has $1+n_{i}$ tab positions. Each piece $P_{i}$ has a tab of full size $n$ at its lowest indexed tab position, i.e., at position $i$. Hence $P_{i}$ has a tab vertex $t_{i}\left(P_{i}\right)=w_{2 i}$. For $i<j \leq i+n_{i}$, the existence and location of the tab vertex $t_{j}\left(P_{i}\right)$ of tab $T_{j}\left(P_{i}\right)$ depends on the size $s_{j}\left(P_{i}\right)$ assigned to tab $T_{j}\left(P_{i}\right)$.

The idea behind the construction is as follows. Realize a depth first search tree for $G$ by polygonal pieces floating parallel to the $x, y$-plane. Arrange these pieces so that the piece $P(v)$ representing a vertex $v$ lies above the pieces representing vertices in the subtree rooted at $v$, with the $x, y$-projection of $P(v)$ containing exactly the projections of the pieces $P(w)$ for which $w$ belongs to the 
subtree rooted at $v$. Thus each piece has the possibility of seeing its ancestors and descendants, but nothing else.

Unless $G$ itself is a tree, depth first search discovers back edges, i.e., edges of $G$ that do not appear as tree edges in the depth first search tree. A familiar property of depth first search trees for graphs is that each back edge must connect an ancestor, descendant pair in the tree. The purpose of adding tabs of varying sizes is to control which ancestors and descendants see each other.
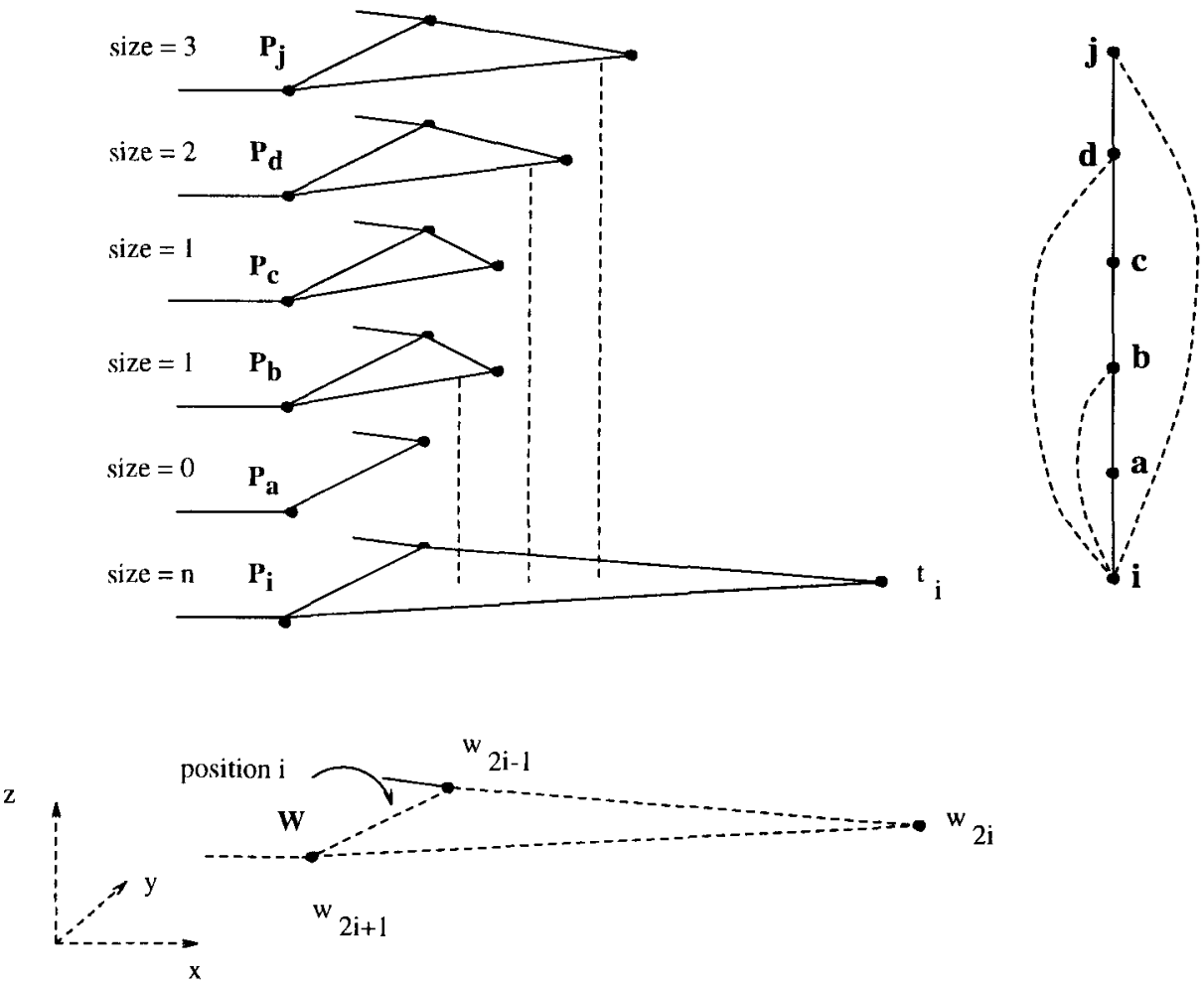

Fig. 9. Back edges from $i$ and their inverted staircase of tabs.

Suppose the depth first search tree has a back edge between $i$ and ancestor $j$ of $i$. Our construction creates a visibility between the tab $T_{i}$ of full size $n$ in position $i$ on $P_{i}$ and a tab in position $i$ on $P_{j}$. See Fig. 9.

Of course there may be back edges in the tree joining $i$ to $k$, where $k$ lies on the path from $i$ to its ancestor $j$. (Consider $k=b, c, d$ in the figures.) In this case, our construction creates a visibility between the tab in position $i$ on $P_{k}$ and the full sized tab in position $i$ on $P_{i}$. Note that the visibility between the tabs in position $i$ on $P_{k}$ and $P_{j}$ must be blocked if the graph $G$ contains no edge between $j$ and $k$. Hence, for example, the tabs in position $i$ on $P_{b}$ and $P_{j}$ must 
be blocked from seeing each other by intervening tabs.

Blocking inappropriate visibilities between tabs is achieved by creating an inverted staircase of tabs above the tab of full size on $P_{i}$ and the tab in position $i$ on $P_{j}$. The tab on $P_{i}$ has full size $n$. The tab in position $i$ on the piece immediately above $P_{i}$ is assigned size 0 , as this piece sees $P_{i}$ in any case. The tab on the next piece above $P_{i}$ is also assigned size 0 unless there is a back edge from $i$ to the vertex corresponding to this piece; in this case, the tab size is increased to 1 . Tab size remains the same or increases with increasing integer $z$ values. In fact, tab size increases precisely when $P_{i}$ and the piece at the $z$ value in question should be mutually visible. Thus the size of the tab in position $i$ on $P_{j}$ is equal to the number of back edges of the form $i, k$, where $k$ lies on the path from $i$ to $j$ (possibly $k=j$ ).

Lemma 5. Let $G$ be a connected graph. The following assignment of parameters to the piece representing an arbitrary vertex $v$ of $G$ gives a 3-dimensional visibility representation for $G$ :

- $v$ is assigned its depth first search order $i$;

- the index $n_{i}$ of $v$ is set equal to the number of descendants of $v$ in the depth first search tree;

- the tab $T_{i}\left(P_{i}\right)$ in position $i$ on $P_{i}$ is assigned size $s_{i}\left(P_{i}\right)=n$;

- for $i<j \leq i+n_{i}$ the size $s_{j}\left(P_{i}\right)$ of the tab $T_{j}\left(P_{i}\right)$ on $P_{i}$ at position $j$ is set equal to the number of nodes on the tree path from $j$, up to and including $i$, that receive a back edge from $j$; and

- the $z$ coordinate of $P_{i}$ is set equal to 1 less than the $z$ coordinate of its parent.

Proof. (Sketch) A well-known property of depth first search ordering is that the descendants of $v$ are numbered with consecutive integers, beginning with $i+1$. Thus $P_{i}$ has, in addition to a tab of full size at position $i$, a tab in position $j$ for $1<j \leq i+n_{i}$.

It is easy to check that the pieces have disjoint interiors and that $P_{i}$ cannot see any $P_{k}$ representing a vertex $w$ unless $w$ is either an ancestor or a descendant of $v$. Clearly, $P_{i}$ sees its parent (if any) and all of its children.

Let us check that if the depth first search tree has a back edge from $v$, where $v$ is numbered $i$, to some ancestor $u$ of $v$, where $u$ is numbered $k$, then $P_{i}$ and $P_{k}$ are mutually visible. $P_{k}$ has a tab in position $i$. This tab aligns with the tab of full size in position $i$ on $P_{i}$. Furthermore, the tab on $P_{k}$ has size greater than the intervening tabs in position $i$, as the number of back edges from $i$ on the path from $i$ to $k$ is at least one greater than the number of back edges on the path from $i$ to $k$, up to but not including $k$. Hence $P_{i}$ and $P_{k}$ have a line of visibility between their tabs at position $i$. Thus all back edges are represented.

It can also be checked that no inappropriate visibilities are present.

It is straightforward to design an algorithm that, by computing tab sizes efficiently, carries out the construction in $O\left(n^{2}\right)$ time. Summarizing we obtain

Theorem 6. Every graph on $n$ vertices is $2 n$-representable. Furthermore, a representation can be constructed in $O\left(n^{2}\right)$ time. 
Corollary 7. The construction of Lemma 5 can be modified to produce convex pieces, fat pieces, polyhedral pieces, or pieces having any combination of these properties.

Proof. To produce convex pieces, use a $W$ with sufficiently many vertices (12n) that each piece has a vertex angle at $O$ of at most $\pi / 6$. To produce fat pieces, move the vertex at $O$ sufficiently close to the chord through the first and last vertices of $P_{i}$ shared with $W$. To produce polyhedral pieces, take the cross product of $P_{i}$ with a short line segment parallel to the $z$ axis.

\section{References}

[A] N. Alon, "The number of polytopes, configurations and real matroids", Mathematika 33 (1986), pp. 62-71

[BEF+] P. Bose, H. Everett, S. Fekete, A. Lubiw, H. Meijer, K. Romanik, T. Shermer and $S$. Whitesides, "On a visibility representation for graphs in three dimensions," in Snapshots of Computational and Discrete Geometry, v. 3, eds. D. Avis and P. Bose, McGill University School of Computer Science Technical Report SOCS-94.50, July 1994, pp. 2 - 25

[CDR] J. Canny, B. Donald and E. K. Ressler, "A rational rotation method for robust geometric algorithms", Proc. 8th ACM Symp. Comput. Geom., 1992, 251 - 260.

[DETT] G. Di Battista, P. Eades, R. Tamassia and I. Tollis, "Algorithms for drawing graphs: an annotated bibliography", Computational Geometry Theory and Applications, v. 4, 1994, 235 - 282. Also available from wilma.cs.brown.edu by ftp.

[DH] A. Dean and J. Hutchison, "Rectangle-visibility representations of bipartite graphs," in Proc. Graph Drawing '94, Princeton, NJ, 1994. Lecture Notes in Computer Science LNCS v. 894, Springer-Verlag, 1995.

[ES] P. Erdös, Gy. Szekeres, "A Combinatorial Problem in Geometry", Compositio Math. 2, 1935, 463-470.

[F] S. Felsner, personal communication, 1995.

[FHW] S. Fekete, M. Houle and S. Whitesides, "New results on a visibility representation of graphs in 3D," in Proc. Symposium on Graph Drawing (GD '95), Passau, Germany, 1995, Springer-Verlag LNCS series (these proceedings).

[G] B. Grünbaum, "On a Conjecture of H. Hadwiger", Pacific J. Math., 11, 215-219.

[H] H. Hadwiger, "Über Treffanzahlen bei translationsgleichen Eikörpern", Arch. Math., Vol. VIII, 1957, 212-213.

[KKU] E. Kranakis, D. Krizanc and J. Urrutia, "On the number of directions in visibility representations of graphs," in Proc. Graph Drawing '94, Princeton, NJ, 1994. Lecture Notes in Computer Science LNCS v. 894, Springer-Verlag, 1995, $167-176$.

[Rom] K. Romanik, "Directed VR-representable graphs have unbounded dimension," in Proc. Graph Drawing '94, Princeton, NJ, 1994. Lecture Notes in Computer Science LNCS v. 894, Springer-Verlag, 1995, 177 - 181.

[TT] R. Tamassia and I. Tollis, "A unified approach to visibility representations of planar graphs," Discrete Comput. Geom. v. 1, 1986, 321 - 341.

[W] S. Wismath, "Characterizing bar line-of-sight graphs," in Proc. ACM Symp. on Computational Geometry, 1985, 147 - 152. 